\title{
Non-gravitational Effects of the Metric Field over Complex Manifolds
}

Swagatam Sen ( $\nabla$ swagatam.x.sen@gmail.com )

https://orcid.org/0000-0002-4336-9196

\section{Research Article}

Keywords: Complex manifold, Geodesic, Gravity, Lorentz Field

Posted Date: January 29th, 2021

DOl: https://doi.org/10.21203/rs.3.rs-163617/v1

License: (c) (i) This work is licensed under a Creative Commons Attribution 4.0 International License. Read Full License 


\title{
NON-GRAVITATIONAL EFFECTS OF THE METRIC FIELD OVER COMPLEX MANIFOLDS
}

\author{
SWAGATAM SEN
}

\begin{abstract}
Aвstract. Objective of this work is to study whether some of the known nongravitational phenomena can be explained as motion on a straight line as gravity is treated within General Relativity. To do that, we explore a metric field on a complexified manifold with holomorphic coordinates. Specifically we look into the behaviour of geodesics on such a smooth complex manifold and the path traced out by its real component. This yields a family of equations of motions in real coordinates which is shown to have deviations from usual geodesic equation and in that way expands the geodesic to capture contributions from additional fields and interactions beyond the mere gravitational ones as a function of the metric field.
\end{abstract}

\section{InTRODUCTION}

General Relativity provides a description of the universe exclusively as a function of the metric field[1][2][3]. It can alternatively be formulated in terms of the connection [4] [5], but that doesn't deter from the purely geometric nature of the theory. In other words, a body subject to solely gravitation field merely follows a straight line on an underlying curved space, irrespective of the properties of the said object [6][7]. However, other known fundamental forces of nature don't share such a geometric description solely based on the metric field. Quantum Field Theory - arguably the best known descriptions of such forces - essentially deals with them as extrinsic non-metric field contributions in the Lagrangian[8][9][10]. In contrast, present work endeavours to study some of the non-gravitational effects of the metric field on its own. However, the field in consideration would be a hermitian metric on a complexified spacetime.

Motivations behind studying complex manifolds within the realms of gravitational physics is a topic that has drawn significant attention since the days of formulation of General Relativity [11][12][13]. Einstein himself had progressed to consider a complex hermitian metric to unify gravity and electromagnetism, albeit

Date: Jan 10, 2021.

2010 Mathematics Subject Classification. Primary 53Z05; Secondary 57N16, 83D05.

Key words and phrases. Complex manifold, Geodesic, Gravity, Lorentz Field. 
defined on real 4-dimensional coordinates [14] 115]. Much before that similar proposals for a complex metric was put forward by Weyl[16][17] and Soh[18] among others. As an approach, it doesn't provide adequately strong structure to conceptually integrate the real and imaginary parts of the metric. For example, under a generic continuous transformation both parts of the metric behave quite independently. That line of investigation has persisted over time[19][20] [21] [22], albeit in a much reduced capacity. We have a fundamental weakness in these approaches which has restricted its successes, because of the metric being considered as function of exclusively real coordinates, or in other words, the framework used in these studies are that of a real manifold with a complex vector bundle structure around it. It doesn't draw in the stronger structure of complex metric defined on a complex manifold.

In a way, that same limitation can be attributed to the standard mathematical approach developed later for Quantum Mechanics, wherein obsevables are conceptualised as linear operators on a complex Hilbert space, which in turn is defined on real phase space coordinates for a quantum system[23]. Consequently the underlying mathematical structure for quantum mechanics developed through numerous groundbreaking work [24][25], while tremendously accurate, appears to be incomplete in addressing the longstanding foundational challenges - that of interpretation [26] [27][28], and that of measurement [29][30][31] among others.

It was primarily through the development of Twistor theory by Penrose [32], that the idea of a complex underlying space to describe the reality branched out into an area of sustained focus. Essentially within Twistor theory, 'real' physical fields are represented as complex objects defined on complex projective space, called Twistor space [33]. Mathematically, that approach offers a much richer structure to work with using contour integral formalism[34] [35], and finds deeper connections with other formalisms like String theory [36] 37].

In the present work we study straight-lines/geodesics of a complexified manifold and the path generated by the real part of that geodesic. Clearly, such path would deviate from a geodesic as calculated on the Riemannian sub-manifold generated by the real component of the bigger complex manifold. In plainer terms, a straightline on the complex manifold would appear as curved to an observer confined on the real submanifold. It is in such deviations, that we hope to recover some of the non-gravitational effects of the metric field.

In the next few sections we would add rigour to that intuitive idea. Throughout we would work with a complex n-manifold $\mathscr{M}$ and its projective real submanifold $\mathscr{R} \subset \mathscr{M}$. Our objective would be to derive the geodesic on the complex manifold 
first using the usual variational technique. Then we would study how that geodesic maps to a curve on the projective sub-manifold $\mathscr{R}$. We would refer this as the projective real geodesic on $\mathscr{R}$ and analyse how that differs from the true geodesic on $\mathscr{R}$.

Definition 1.1. For a complex n-manifold $\mathscr{M}$ with a holomorphic atlas $\{(z, U)\}, \mathscr{R} \subset$ $\mathscr{M}$ is a projective real sub-manifold if $\forall$ holomorphic charts $(z, U)$ on $\mathscr{M}, \exists$ a projection $\pi$ : $\mathscr{M} \mapsto \mathscr{R}$ and a continuous chart $\chi: \pi(U) \mapsto \mathbb{R}^{n}$ such that $\chi \circ \pi=\operatorname{Re}(z)$

This would allow us to write down the local coordinates as $z^{\alpha}=x^{\alpha}+i t^{\alpha}$ and $\bar{z}^{\beta}=x^{\beta}-i t^{\beta}$ where $x=\chi \circ \pi$ and $t=\operatorname{Im}(z)$.

Next we would need to introduce a hermitian metric $g$ on $\mathscr{M}$ with the usual distance 2-form in local coordinates,

$$
d \tau^{2}=g_{\alpha \bar{\beta}} d z^{\alpha} \overline{d z^{\beta}}
$$

Our end goal in the next section would be to derive a geodesic equation using the complex/conjugate coordinates which is summarised in Theorem 2.2. Then in Theorem 2.5, we would further prove that this geodesic allows a projective geodesic on the real coordinates which has the generic structural form as below,

$$
h_{\mu \gamma} D^{2} x^{\mu}=\Upsilon_{\gamma \alpha \beta}^{(1,1)} D x^{\alpha} D x^{\beta}-\Upsilon_{\gamma \alpha \beta}^{(1,0)} D x^{\beta} D t^{\alpha}-\Upsilon_{\gamma \alpha \beta}^{(0,0)} D t^{\alpha} D t^{\beta}
$$

for some tensor $h$ and symbols $\Upsilon^{(0,0)}, \Upsilon^{(1,0)}, \Upsilon^{(1,1)}$ all of which are functions of the metric.

These theorems would then allow us to consider certain known special cases and illustrate how equations of motions for gravitational and Lorentz Fields can naturally manifest from Theorem 2.5 as first order terms under special configurations. Not only that we would briefly discuss the implications of the infinite series of higher order terms in the equation, which contain information about how real and imaginary parts of the metric interact. Intuition behind such investigations lies in the realisation that the real symmetric part of the metric represent matter and gravitational field in the usual way of GR, while the antisymmetric imaginary part represent the EM field. With that backdrop the infinitely additive interaction terms would represent the interactions of the gravitation and electromagnetic fields at various orders. 


\section{Results}

To achieve the initial goal of deriving the geodesic on the complex manifold using complex/conjugate coordinates, we would follow the usual approach of variational calculus.

Under the metric $g$, the arc length for a given path parametrised by $\sigma \in[0,1]$, would be $\tau=\int_{0}^{1} L\left(\mathbf{z}, \frac{d \mathbf{z}}{d \sigma}\right) d \sigma$, where

$$
L=\sqrt{g_{\alpha \bar{\beta}} \frac{d z^{\alpha}}{d \sigma} \frac{d \overline{z^{\beta}}}{d \sigma}}
$$

is the Lagrangian for equation of motion.

Note. Since $g$ is hermitian, $L$ is real and we can think of minimising the arc length in the usual variational approach.

Note. the path can be re-parametrized using $\tau$ instead of arbitrary $\sigma$ and the derivatives can be replaced as $\frac{d}{d \sigma}=L \frac{d}{d \tau}$. Notationally henceforth we would use $D=$ $\frac{d}{d \tau}$.

Proposition 2.1. Let $D z^{\gamma}=\frac{d z^{\gamma}}{d \tau}$ and $D \bar{z}^{\gamma}=\frac{d \bar{z}^{\gamma}}{d \tau}$ be path derivatives of the complex and conjugate coordinates. Also let $\partial_{\alpha}=\frac{\partial}{\partial z^{\alpha}}$ and $\partial_{\bar{\beta}}=\frac{\partial}{\partial \overline{z^{\beta}}}$ be the partial derivatives with respect to complex and conjugate coordinates. Then,

$$
\frac{d}{d \sigma}\left[\frac{\partial L}{\partial\left(d \overline{z^{\gamma}} / d \sigma\right)}\right]=\frac{L}{2}\left[\partial_{\beta} g_{\alpha \bar{\gamma}} D z^{\alpha} D z^{\beta}+\partial_{\bar{\beta}} g_{\alpha \bar{\gamma}} D z^{\alpha} D \bar{z}^{\beta}+g_{\alpha} \bar{\gamma} D^{2} z^{\alpha}\right]
$$

Proof. From definition of $L$ we know that,

$$
\frac{\partial L}{\partial\left(d \overline{z^{\gamma}} / d \sigma\right)}=\frac{1}{2 L} g_{\alpha} \bar{\gamma} \frac{d z^{\alpha}}{d \sigma}
$$

That, in turn, would allow us to write,

$$
\begin{aligned}
\frac{d}{d \sigma} \frac{\partial L}{\partial\left(d \overline{z^{\gamma}} / d \sigma\right)} & =\frac{d}{d \sigma} \frac{1}{2 L} g_{\alpha \bar{\gamma}} \frac{d z^{\alpha}}{d \sigma}=\frac{L}{2} \frac{d}{d \tau} g_{\alpha \bar{\gamma}} \frac{d z^{\alpha}}{d \tau} \\
& =\frac{L}{2}\left[\frac{d g_{\alpha \bar{\gamma}}}{d \tau} \frac{d z^{\alpha}}{d \tau}+g_{\alpha \bar{\gamma}} \frac{d^{2} z^{\alpha}}{d \tau^{2}}\right] \\
& =\frac{L}{2}\left[\partial_{\beta} g_{\alpha \bar{\gamma}} \frac{d z^{\alpha}}{d \tau} \frac{d z^{\beta}}{d \tau}+\partial_{\bar{\beta}} g_{\alpha \bar{\gamma}} \frac{d z^{\alpha}}{d \tau} \frac{d \overline{z^{\beta}}}{d \tau}+g_{\alpha \bar{\gamma}} \frac{d^{2} z^{\alpha}}{d \tau^{2}}\right]
\end{aligned}
$$


We can now use this result to derive the geodesic equation in complex and conjugate coordinates.

Theorem 2.2. Let $\mathscr{M}$ be a complex manifold with a hermitian metric $g$. If $\tau$ is the cumulative length of a given path $\Omega$ on $\mathscr{M}$, then $\Omega$ is a geodesic iff

$$
g_{\mu \bar{\gamma}} D^{2} z^{\mu}=\left(\partial_{\bar{\gamma}} g_{\alpha \bar{\beta}}-\partial_{\bar{\beta}} g_{\alpha \bar{\gamma}}\right) D z^{\alpha} D \overline{z^{\beta}}-\partial_{\alpha} g_{\beta \bar{\gamma}} D z^{\alpha} D z^{\beta}
$$

Proof. We'd first note that,

$$
\frac{\partial L}{\partial \overline{z^{\gamma}}}=\frac{L}{2} \partial_{\bar{\gamma}} g_{\alpha \bar{\beta}} D z^{\alpha} D \overline{z^{\beta}}
$$

Now the result follows from a direct application of the Euler-Lagrange Eqn on conjugate coordinates

$$
\frac{\partial L}{\partial \overline{z^{\gamma}}}=\frac{d}{d \sigma}\left[\frac{\partial L}{\partial\left(d \overline{z^{\gamma}} / d \sigma\right)}\right]
$$

and replacing the right hand side of the equation using Proposition 2.1

It's easy to see that Theorem 2.2 is a direct generalisation of geodesic on real coordinates. However, as it can be expected, if one extracts the real component $D^{2} x^{\mu}$ from the complex geodesic equation, it would contain structurally additional components as functions of real and imaginary parts of the metric as well as first order derivatives of $x$ and $t$ coordinates.

But before we introduce such a Projective geodesic on real coordinates, we'd need to introduce a set of objects that would serve the purpose of connection coefficients.

Definition 2.1. For a hermitian metric $g=g^{R}+i g^{I}$ on a Complex manifold $\mathscr{M}$, we define Normal Field as the combination

$$
\Phi=\left(\Phi_{\alpha \beta}^{\gamma++}, \Phi_{\alpha \beta}^{\gamma+-}, \Phi_{\alpha \beta}^{\gamma-+}, \Phi_{\alpha \beta}^{\gamma--}\right)
$$

where

- $\Phi_{\alpha \bar{\beta}}^{\gamma++}=\frac{1}{2}\left[\partial_{\gamma}^{x} g_{\alpha \bar{\beta}}^{R}-\partial_{\alpha}^{x} g_{\beta \bar{\gamma}}^{R}-\partial_{\beta}^{x} g_{\alpha \bar{\gamma}}^{R}\right]$

- $\Phi_{\alpha \bar{\beta}}^{\gamma+-}=\frac{1}{2}\left[\partial_{\gamma}^{x} g_{\alpha \bar{\beta}}^{I}-\partial_{\alpha}^{x} g_{\beta \bar{\gamma}}^{I}-\partial_{\beta}^{x} g_{\alpha \bar{\gamma}}^{I}\right]$

- $\Phi_{\alpha \bar{\beta}}^{\gamma-+}=\frac{1}{2}\left[\partial_{\gamma}^{t} g_{\alpha \bar{\beta}}^{R}-\partial_{\alpha}^{t} g_{\beta \bar{\gamma}}^{R}-\partial_{\beta}^{t} g_{\alpha \bar{\gamma}}^{R}\right]$

- $\Phi_{\alpha \bar{\beta}}^{\gamma--}=\frac{1}{2}\left[\partial_{\gamma}^{t} g_{\alpha \bar{\beta}}^{I}-\partial_{\alpha}^{t} g_{\beta \bar{\gamma}}^{I}-\partial_{\beta}^{t} g_{\alpha \bar{\gamma}}^{I}\right]$

are the ordinary Christoffel symbols for real and imaginary parts of the metric in real and imaginary coordinates respectively.

Note. If $g$ is real symmetric, then $\Phi_{\alpha \beta}^{\gamma+-}=\Phi_{\alpha \beta}^{\gamma--}=0$ 
Note. If $g$ is independent of $t$, then $\Phi_{\alpha \beta}^{\gamma-+}=\Phi_{\alpha \beta}^{\gamma--}=0$

Note. $\Phi_{\alpha \alpha}^{\gamma+-}=\Phi_{\alpha \alpha}^{\gamma--}=0$

Proposition 2.3. For a hermitian metric $g=g^{R}+i g^{I}$ with Normal Field $\Phi$, we can write,

(1) $\partial_{\bar{\gamma}} g_{\alpha \bar{\beta}}-\partial_{\bar{\beta}} g_{\alpha \bar{\gamma}}-\partial_{\alpha} g_{\beta \bar{\gamma}}=\Phi_{\alpha \bar{\beta}}^{\gamma++}+i \Phi_{\alpha \bar{\beta}}^{\gamma+-}+\frac{i}{2} \partial_{\gamma}^{t} g_{\alpha \bar{\beta}}^{R}$

(2) $\partial_{\bar{\gamma}} g_{\alpha \bar{\beta}}-\partial_{\bar{\beta}} g_{\alpha \bar{\gamma}}+\partial_{\alpha} g_{\beta \bar{\gamma}}=\frac{1}{2} \partial_{\gamma}^{x} g_{\alpha \bar{\beta}}^{R}-\Phi_{\alpha \bar{\beta}}^{\gamma ;--}+i \Phi_{\alpha \bar{\beta}}^{\gamma-+}$

Proof. As we know,,

$$
\begin{aligned}
\partial_{\bar{\gamma} \bar{\gamma} g_{\bar{\beta}}}-\partial_{\bar{\beta}} g_{\alpha \bar{\gamma}}=\frac{1}{2}\left[\partial_{\gamma}^{x} g_{\alpha \bar{\beta}}^{R}-\partial_{\gamma}^{t} g_{\alpha \bar{\beta}}^{I}\right. & \left.-\partial_{\beta}^{x} g_{\alpha \bar{\gamma}}^{R}+\partial_{\beta}^{t} g_{\alpha \bar{\gamma}}^{I}\right] \\
& +\frac{i}{2}\left[\partial_{\gamma}^{x} g_{\alpha \bar{\beta}}^{I}+\partial_{\gamma}^{t} g_{\alpha \bar{\beta}}^{R}-\partial_{\beta}^{x} g_{\alpha \bar{\gamma}}^{I}-\partial_{\beta}^{t} g_{\alpha \bar{\gamma}}^{R}\right]
\end{aligned}
$$

we can write,

$$
\begin{aligned}
\partial_{\bar{\gamma}} g_{\alpha \bar{\beta}}-\partial_{\bar{\beta}} g_{\alpha \bar{\gamma}}-\partial_{\alpha} g_{\beta \bar{\gamma}} & =\frac{1}{2}\left[\partial_{\gamma}^{x} g_{\alpha \bar{\beta}}^{R}-\partial_{\beta}^{x} g_{\alpha \bar{\gamma}}^{R}-\partial_{\alpha}^{x} g_{\bar{\beta} \bar{\gamma}}^{R}\right] \\
& +\frac{i}{2}\left[\partial_{\gamma}^{x} g_{\alpha \bar{\beta}}^{I}-\partial_{\beta}^{x} g_{\alpha \bar{\gamma}}^{I}-\partial_{\alpha}^{x} g_{\beta \bar{\gamma}}^{I}\right]+\frac{i}{2} \partial_{\gamma}^{t} g_{\alpha \bar{\beta}}^{R} \\
& =\Phi_{\alpha \bar{\beta}}^{\gamma++}+i \Phi_{\alpha \bar{\beta}}^{\gamma+-}+\frac{i}{2} \partial_{\gamma}^{t} g_{\alpha \bar{\beta}}^{R}
\end{aligned}
$$

Similarly,

$$
\begin{aligned}
\partial_{\bar{\gamma} g_{\alpha \bar{\beta}}-\partial_{\bar{\beta}} g_{\alpha \bar{\gamma}}+\partial_{\alpha} g_{\beta \bar{\gamma}}} & =\frac{1}{2} \partial_{\gamma}^{x} g_{\alpha \bar{\beta}}^{R}-\frac{1}{2}\left[\partial_{\gamma}^{t} g_{\alpha \bar{\beta}}^{I}-\partial_{\beta}^{t} g_{\alpha \bar{\gamma}}^{I}-\partial_{\alpha}^{t} g_{\beta \bar{\gamma}}^{I}\right] \\
& +\frac{i}{2}\left[\partial_{\gamma}^{t} g_{\alpha \bar{\beta}}^{R}-\partial_{\beta}^{t} g_{\alpha \bar{\gamma}}^{R}-\partial_{\alpha}^{t} g_{\beta \bar{\gamma}}^{R}\right] \\
& =-\Phi_{\alpha \bar{\beta}}^{\gamma--}+i \Phi_{\alpha \bar{\beta}}^{\gamma-+}+\frac{1}{2} \partial_{\gamma}^{x} g_{\alpha \bar{\beta}}^{R}
\end{aligned}
$$

Definition 2.2. For a hermitian metric $g=g^{R}+i g^{I}$ on a Complex manifold $\mathscr{M}$, we define Auxiliary Field as

$$
F=\left(F_{\alpha \gamma \beta}^{x}, F_{\alpha \gamma \beta}^{t}\right)
$$

where

- $F_{\alpha \gamma \beta}^{x}=\partial_{\gamma}^{x} g_{\alpha \bar{\beta}}^{I}-\partial_{\beta}^{x} g_{\alpha \bar{\gamma}}^{I}$

- $F_{\alpha \gamma \beta}^{t}=\partial_{\gamma}^{t} g_{\alpha \bar{\beta}}^{I}-\partial_{\beta}^{t} g_{\alpha \bar{\gamma}}^{I}$

Note. Auxiliary Field symbols are anti-symmetric in $\beta, \gamma$ 
Note. If $g$ is real symmetric, then $F_{\alpha \gamma \beta}^{x}=F_{\alpha \gamma \beta}^{t}=0$

Note. Auxiliary Field symbols are covariant 2-tensors in $\beta, \gamma$ indices under holomorphic coordinate changes.

Proposition 2.4. For a hermitian metric $g=g^{R}+i g^{I}$ with Auxiliary field F, we can write,

$$
2 \partial_{\bar{\gamma}} g_{\alpha \bar{\beta}}^{I}+i \partial_{\beta}^{x} g_{\alpha \bar{\gamma}}+\partial_{\alpha}^{t} g_{\beta \bar{\gamma}}=F_{\alpha \gamma \beta}^{x}+\partial_{\alpha}^{t} g_{\beta \bar{\gamma}}^{I}-i F_{\beta \gamma \alpha}^{t}+i \partial_{\beta}^{x} g_{\alpha \bar{\gamma}}^{R}
$$

Proof. Clearly,

$$
\begin{aligned}
2 \partial_{\bar{\gamma}} g_{\alpha \bar{\beta}}^{I} & +i \partial_{\beta}^{x} g_{\alpha \bar{\gamma}}+\partial_{\alpha}^{t} g_{\beta \bar{\gamma}} \\
& =\partial_{\gamma}^{x} g_{\alpha \bar{\beta}}^{I}+i \partial_{\gamma}^{t} g_{\alpha \bar{\beta}}^{I}+i \partial_{\beta}^{x} g_{\alpha \bar{\beta}}^{R}-\partial_{\beta}^{x} g_{\alpha \bar{\gamma}}^{I}+\partial_{\alpha}^{t} g_{\beta \bar{\gamma}}^{R}+i \partial_{\alpha}^{t} g_{\beta \bar{\gamma}}^{I} \\
& =\left(\partial_{\gamma}^{x} g_{\alpha \bar{\beta}}^{I}-\partial_{\beta}^{x} g_{\alpha \bar{\gamma}}^{I}\right)+\partial_{\alpha}^{t} g_{\beta \bar{\gamma}}^{I}-i\left(\partial_{\gamma}^{t} g_{\alpha \bar{\beta}}^{I}-\partial_{\alpha}^{t} g_{\beta \bar{\gamma}}^{I}\right)+i \partial_{\beta}^{x} g_{\alpha \bar{\gamma}}^{R} \\
& =F_{\alpha \gamma \beta}^{x}+\partial_{\alpha}^{t} g_{\beta \bar{\gamma}}^{I}-i F_{\beta \gamma \alpha}^{t}+i \partial_{\beta}^{x} g_{\alpha \bar{\gamma}}^{R}
\end{aligned}
$$

Definition 2.3. For a hermitian metric $g$ on a complex manifold $\mathscr{M}$ with $g=g^{R}+$ $i g^{I}$, we define Coupling tensor as $\epsilon_{\gamma}^{\eta}=g_{v \bar{\gamma}}^{I} g^{R ; v \bar{\eta}}$

Note. If $g$ is real symmetric then $\epsilon_{\gamma}^{\eta}=0$

Coupling tensor is a critical component in the interpretation of the theory that we are going to outline next. As described before, coupling tensor represents the relative strength of the EM potential over the gravitational one and is a tensor generalisation of the charge-to-mass ratio. In the subsequent build-up, we would see that it serves as the fundamental vehicle to describe interactions between the two fields.

Theorem 2.5. Let $\mathscr{R}$ be a real projective manifold embedded within a complex manifold $\mathscr{M}$. Let $g$ be a hermitian metric on $\mathscr{M}$ with $g=g^{R}+i g^{I}$. Additionally let $\Phi, F$ be the primary and secondary fields respectively and let $\epsilon$ be the coupling tensor. Then $\mathscr{R}$ is endowed with a real projective geodesic described by

$$
\left(g_{\mu \bar{\gamma}}^{R}+\epsilon_{\gamma}^{\eta} g_{\mu \bar{\eta}}^{I}\right) D^{2} x^{\mu}=\Upsilon_{\gamma \alpha \beta}^{(1,1)} D x^{\alpha} D x^{\beta}-\Upsilon_{\gamma \alpha \beta}^{(1,0)} D x^{\beta} D t^{\alpha}-\Upsilon_{\gamma \alpha \beta}^{(0,0)} D t^{\alpha} D t^{\beta}
$$

where

- $\Upsilon_{\gamma \alpha \beta}^{(1,1)}=\Phi_{\alpha \bar{\beta}}^{\gamma++}+\epsilon_{\gamma}^{\eta} \Phi_{\alpha \bar{\beta}}^{\gamma+-}+\frac{1}{2} \epsilon_{\gamma}^{\eta} \partial_{\eta}^{t} g_{\alpha \bar{\beta}}^{R}$

- $\Upsilon_{\gamma \alpha \beta}^{(1,0)}=F_{\alpha \gamma \beta}^{x}-\epsilon_{\gamma}^{\eta} F_{\beta \eta \alpha}^{t}+\partial_{\alpha}^{t} g_{\beta \bar{\gamma}}^{R}+\epsilon_{\gamma}^{\eta} \partial_{\beta}^{x} g_{\alpha \bar{\eta}}^{R}$

- $\Upsilon_{\gamma \alpha \beta}^{(0,0)}=\Phi_{\alpha \bar{\beta}}^{\gamma--}-\frac{1}{2} \partial_{\gamma}^{x} g_{\alpha \bar{\beta}}^{R}-\epsilon_{\gamma}^{\eta} \Phi_{\alpha \bar{\beta}}^{\gamma-+}$ 
Proof. First of all we can write,

$$
\begin{aligned}
& {\left[\partial_{\bar{\gamma}} g_{\alpha \bar{\beta}}-\partial_{\bar{\beta}} g_{\alpha \bar{\gamma}}\right] D z^{\alpha} D \bar{z}^{\beta}} \\
& =\left[\partial_{\bar{\gamma}} g_{\alpha \bar{\beta}}-\partial_{\bar{\beta}} g_{\alpha \bar{\gamma}}\right]\left[D x^{\alpha} D x^{\beta}+D t^{\alpha} D t^{\beta}+i\left(D t^{\alpha} D x^{\beta}-D t^{\beta} D x^{\alpha}\right)\right] \\
& =\left[\partial_{\bar{\gamma}} g_{\alpha \bar{\beta}}-\partial_{\bar{\beta}} g_{\alpha \bar{\gamma}}\right]\left[D x^{\alpha} D x^{\beta}+D t^{\alpha} D t^{\beta}\right]+i\left[2 \partial_{\bar{\gamma}} g_{\alpha \bar{\beta}}^{I}-\partial_{\bar{\beta}} g_{\alpha \bar{\gamma}}+\partial_{\bar{\alpha}} g_{\beta \bar{\gamma}}\right] D t^{\alpha} D x^{\beta}
\end{aligned}
$$

Also,

$$
\begin{aligned}
\partial_{\beta} g_{\alpha \bar{\gamma}} D z^{\alpha} D z^{\beta} & =\partial_{\beta} g_{\alpha \bar{\gamma}}\left[D x^{\alpha} D x^{\beta}-D t^{\alpha} D t^{\beta}+i\left(D t^{\alpha} D x^{\beta}+D t^{\beta} D x^{\alpha}\right)\right] \\
& =\partial_{\alpha} g_{\beta \bar{\gamma}}\left[D x^{\alpha} D x^{\beta}-D t^{\alpha} D t^{\beta}\right]+i\left[\partial_{\beta} g_{\alpha \bar{\gamma}}+\partial_{\alpha} g_{\beta \bar{\gamma}}\right] D t^{\alpha} D x^{\beta}
\end{aligned}
$$

That implies,

$$
\begin{aligned}
& {\left[\partial_{\bar{\gamma}} g_{\alpha \bar{\beta}}-\partial_{\bar{\beta}} g_{\alpha \bar{\gamma}}\right] D z^{\alpha} D \bar{z}^{\beta}-\partial_{\beta} g_{\alpha \bar{\gamma}} D z^{\alpha} D z^{\beta}} \\
& \quad=\left[\partial_{\bar{\gamma}} g_{\alpha \bar{\beta}}-\partial_{\bar{\beta}} g_{\alpha \bar{\gamma}}-\partial_{\alpha} g_{\beta \bar{\gamma}}\right] D x^{\alpha} D x^{\beta}+\left[\partial_{\bar{\gamma}} g_{\alpha \bar{\beta}}-\partial_{\bar{\beta}} g_{\alpha \bar{\gamma}}+\partial_{\alpha} g_{\beta \bar{\gamma}}\right] D t^{\alpha} D t^{\beta} \\
& \quad-\left[2 \partial_{\bar{\gamma}} g_{\alpha \bar{\beta}}^{I}+i \partial_{\beta}^{x} g_{\alpha \bar{\gamma}}+\partial_{\alpha}^{t} g_{\beta \bar{\gamma}}\right] D t^{\alpha} D x^{\beta}
\end{aligned}
$$

Now using this last equation alongside Theorem 2.2, Propositions 2.3 and 2.4 . we get,

$$
\begin{aligned}
g_{\mu \bar{\gamma}} D^{2} z^{\mu} & =\left[\partial_{\bar{\gamma}} g_{\alpha \bar{\beta}}-\partial_{\bar{\beta}} g_{\alpha \bar{\gamma}}\right] D z^{\alpha} D \bar{z}^{\beta}-\partial_{\beta} g_{\alpha \bar{\gamma}} D z^{\alpha} D z^{\beta} \\
& =\left(\Phi_{\alpha \bar{\beta}}^{\gamma++}+i \Phi_{\alpha \bar{\beta}}^{\gamma+-}+\frac{i}{2} \partial_{\gamma}^{t} g_{\alpha \bar{\beta}}^{R}\right) D x^{\alpha} D x^{\beta}+\left(\frac{1}{2} \partial_{\gamma}^{x} g_{\alpha \bar{\beta}}^{R}-\Phi_{\alpha \bar{\beta}}^{\gamma ;--}+i \Phi_{\alpha \bar{\beta}}^{\gamma-+}\right) D t^{\alpha} D t^{\beta} \\
& -\left(F_{\alpha \gamma \beta}^{x}+\partial_{\alpha}^{t} g_{\beta \bar{\gamma}}^{I}-i F_{\beta \gamma \alpha}^{t}+i \partial_{\beta}^{x} g_{\alpha \bar{\gamma}}^{R}\right) D t^{\alpha} D x^{\beta}
\end{aligned}
$$

But we'd note that,

$$
g_{\mu \bar{\gamma}} D^{2} z^{\mu}=\left(g_{\mu \bar{\gamma}}^{R} D^{2} x^{\mu}-g_{\mu \bar{\gamma}}^{I} D^{2} t^{\mu}\right)+i\left(g_{\mu \bar{\gamma}}^{R} D^{2} t^{\mu}+g_{\mu \bar{\gamma}}^{I} D^{2} x^{\mu}\right)
$$

Equating real and imaginary parts we get the dual identity,

$$
\begin{aligned}
g_{\mu \bar{\gamma}}^{R} D^{2} x^{\mu}-g_{\mu \bar{\gamma}}^{I} D^{2} t^{\mu}=\Phi_{\alpha \bar{\beta}}^{\gamma++} D x^{\alpha} D x^{\beta}+\left(\frac{1}{2} \partial_{\gamma}^{x} g_{\alpha \bar{\beta}}^{R}-\Phi_{\alpha \bar{\beta}}^{\gamma ;--}\right) D t^{\alpha} D t^{\beta} \\
-\left(F_{\alpha \gamma \beta}^{x}+\partial_{\alpha}^{t} g_{\beta \bar{\gamma}}^{I}\right) D t^{\alpha} D x^{\beta}
\end{aligned}
$$

and

$$
\begin{aligned}
g_{\mu \bar{\eta}}^{R} D^{2} t^{\mu}+g_{\mu \bar{\eta}}^{I} D^{2} x^{\mu}=\left(\Phi_{\alpha \bar{\beta}}^{\eta+-}+\frac{1}{2} \partial_{\eta}^{t} g_{\alpha \bar{\beta}}^{R}\right) D x^{\alpha} D x^{\beta} & +\Phi_{\alpha \bar{\beta}}^{\eta-+} D t^{\alpha} D t^{\beta} \\
& -\left(\partial_{\beta}^{x} g_{\alpha \bar{\eta}}^{R}-F_{\beta \eta \alpha}^{t}\right) D t^{\alpha} D x^{\beta}
\end{aligned}
$$


Multiplying the second identity by $\epsilon_{\gamma}^{\eta}$ and adding the identities, we arrive at the desired result.

Next we'd investigate some of the specific ramifications and special cases of Theorem 2.5 through a set of corollaries.

Corollary. [Levi-Civita] Let $\mathscr{M}$ be a 4-dimensional complex manifold with a real symmetric metric $g$ and let $\mathscr{R}$ be a projective real sub-manifold. Additionally let's assume that $\forall \alpha, \beta$,

$$
\partial_{1}^{x} g_{\alpha \bar{\beta}}=\partial_{2}^{t} g_{\alpha \bar{\beta}}=\partial_{3}^{t} g_{\alpha \bar{\beta}}=\partial_{4}^{t} g_{\alpha \bar{\beta}}=0
$$

Then the projective geodesic is identical to the geodesic on $\mathscr{R}$ under a Levi-Civita connection.

Proof. Proof follows by considering $g^{I}=0$ and setting the specific partial derivatives to 0 , in which case Theorem 2.5 simplifies to

$$
D^{2} x^{\mu}=g^{\mu \gamma} \Phi_{\alpha \beta}^{\gamma++} D x^{\alpha} D x^{\beta}-g^{\mu \gamma} \partial_{1}^{t} g_{\beta \gamma} D t^{1} D x^{\beta}-\frac{1}{2} g^{\mu \gamma} \partial_{\gamma}^{x} g_{11}\left(D t^{1}\right)^{2}
$$

where $\alpha, \beta, \mu>1$ This can be rewritten as

$$
D^{2} y^{\mu}=-\Gamma_{a b}^{\mu} D y^{a} D y^{b}
$$

where $a, b>0, \mu>1$ and $y=\left(t^{1}, x^{2}, x^{3}, x^{4}\right)$. $\Gamma$ denotes the usual Christoffel symbols and the equation precisely signifies the classical geodesic path.

We'd refer the quantity $G^{\mu}=-\Gamma_{a b}^{\mu} D y^{a} D y^{b}$ as the Gravitation field. Indeed Corollary 2 cements a notion that we have alluded to previously, that the real part of the metric represents the symmetric gravitational potential.

It's interesting to note that Theorem 2.5 doesn't prescribe a single equation for $D^{2} x^{\mu}$. Rather it describes a family of geodesics parametrised by the extrinsic parameters $D t^{\alpha}$. This is a point of deviation from the geodesics of GR where the 'straight line' is defined via a single universal differential equation. Instead here we have a family of equations with some extrinsic parameters. One particular choice of such parameters and consequentially, geodesics, would be the Root Geodesics, where $D t^{\alpha}=1, \forall \alpha$

Corollary (Lorentz Field). Let $\mathscr{M}$ be a 4-dimensional complex manifold with a hermitian metric $g$ with $\epsilon_{\gamma}^{\eta} \rightarrow 0$. Also let $\mathscr{R}$ be a projective real sub-manifold. Additionally let's assume that $\forall \alpha, \beta$,

$$
\partial_{1}^{x} g_{\alpha \bar{\beta}}=\partial_{2}^{t} g_{\alpha \bar{\beta}}=\partial_{3}^{t} g_{\alpha \bar{\beta}}=\partial_{4}^{t} g_{\alpha \bar{\beta}}=0
$$


Then the projective root geodesic is given by,

$$
D^{2} x^{\mu}=G^{\mu}+L^{\mu}
$$

where

$$
L^{\mu}=-g^{R ; \mu \bar{\gamma}} F_{1 \gamma \beta}^{x} D x^{\beta}-g^{R ; \mu \bar{\gamma}} \Phi_{1 \overline{1}}^{\gamma--}
$$

Proof. Proof follows by admitting the limit $\epsilon_{\gamma}^{\eta} \rightarrow 0$ and allowing $D t^{1}=1$ in Theorem 2.5

$$
\begin{aligned}
g_{\mu \bar{\gamma}}^{R} D^{2} x^{\mu} & =\Phi_{\alpha \beta}^{\gamma++} D x^{\alpha} D x^{\beta}-\partial_{1}^{t} g_{\beta \gamma} D x^{\beta}-\frac{1}{2} g^{\mu \gamma} \partial_{\gamma}^{x} g_{11} \\
& -F_{1 \gamma \beta}^{x} D x^{\beta}-\Phi_{1 \overline{1}}^{\gamma--} \\
& =g_{\mu \bar{\gamma}}^{R} G^{\gamma}-F_{1 \gamma \beta}^{x} D x^{\beta}-\Phi_{1 \overline{1}}^{\gamma--}
\end{aligned}
$$

$L^{\mu}$ would be referred as the Lorentz field. Indeed when $g_{\mu \bar{\gamma}}^{R}=\delta_{\mu \gamma}$ is Euclidean, we have a familiar form of a Lorenz field,

$$
L^{\mu}=-F_{1 \gamma \beta}^{x} D x^{\beta}-\Phi_{1 \overline{1}}^{\gamma--}
$$

where $F_{1 \gamma \beta}^{x}$ represents anti-symmetric Magnetic tensor and $\Phi_{1 \overline{1}}^{\gamma--}$ represents Electric field. In fact, since,

$$
F_{1 \gamma \beta}^{x}=\partial_{\gamma}^{x} g_{1 \bar{\beta}}^{I}-\partial_{\beta}^{x} g_{1 \bar{\gamma}}^{I}
$$

naturally we can characterise $A_{\gamma}=g_{1 \bar{\gamma}}^{I}$ as the magnetic potential. If we allow $\phi$ such that $\partial_{\gamma}^{x} \phi=\Phi_{11}^{\gamma--}$ then we can write $\left(\phi, A_{2}, A_{3}, A_{4}\right)$ as the 4 -potential for the field.

In a more general case when $\epsilon_{\gamma}^{\eta}$ isn't insignificant but still $\|\epsilon\|<1$, we can rewrite Theorem 2.5 for $D^{2} x^{\mu}$ as an infinite sum of perturbations involving increasing powers of the coupling tensor.

Corollary (Weak Coupling). With slight abuse of notation let $g^{R}, g^{I}$ and $\epsilon$ denote the matrix representation of $g_{\mu \bar{\gamma}}^{R}, g_{\mu \bar{\gamma}}^{I}$ and $\epsilon_{\gamma}^{\eta}$. If $\left\|\epsilon^{2}\right\|<1$ then

$$
D^{2} \mathbf{x}=\left[I+\sum_{k=1}^{\infty}(-1)^{k} \epsilon^{2 k}\right]\left(g^{R}\right)^{-1} \Upsilon
$$

where $\Upsilon$ is a vector shorthand with elements

$$
\Upsilon_{\gamma}=\Upsilon_{\gamma \alpha \beta}^{(1,1)} D x^{\alpha} D x^{\beta}-\Upsilon_{\gamma \alpha \beta}^{(1,0)} D x^{\beta} D t^{\alpha}-\Upsilon_{\gamma \alpha \beta}^{(0,0)} D t^{\alpha} D t^{\beta}
$$


Proof. Proof is trivial as it involves inverting the matrix $\left(g^{R}+g^{I} \epsilon\right)$ as

$$
\left(g^{R}+g^{I} \epsilon\right)^{-1}=\left[g^{R}\left(I+\epsilon^{2}\right)\right]^{-1}=\left[I+\sum_{k=1}^{\infty}(-1)^{k} \epsilon^{2 k}\right]\left(g^{R}\right)^{-1}
$$

Finally the only remaining case of interest is the one of strong coupling where $\left\|\epsilon^{2}\right\| \geq 1$.

Corollary (Strong Coupling). Continuing with the matrix representation $g^{R}, g^{I}$ and $\epsilon$ we can write if $\left\|\epsilon^{2}\right\| \geq 1$

$$
D^{2} \mathbf{x}=\left[I+\sum_{k=1}^{\infty}(-1)^{k} \epsilon^{-2 k}\right] \epsilon^{-2}\left(g^{R}\right)^{-1} \Upsilon
$$

where $\Upsilon$ denoting as usual the vector shorthand with elements

$$
\Upsilon_{\gamma}=\Upsilon_{\gamma \alpha \beta}^{(1,1)} D x^{\alpha} D x^{\beta}-\Upsilon_{\gamma \alpha \beta}^{(1,0)} D x^{\beta} D t^{\alpha}-\Upsilon_{\gamma \alpha \beta}^{(0,0)} D t^{\alpha} D t^{\beta}
$$

Proof. As before the proof follows from Theorem 2.5 by inverting the matrix $\left(g^{R}+\right.$ $\left.g^{I} \epsilon\right)$ but in a slightly different way. Proof is trivial as it involves inverting the matrix $\left(g^{R}+g^{I} \epsilon\right)$ as

$$
\left(g^{R}+g^{I} \epsilon\right)^{-1}=\left[g^{R} \epsilon^{2}\left(I+\epsilon^{-2}\right)\right]^{-1}=\left[I+\sum_{k=1}^{\infty}(-1)^{k} \epsilon^{-2 k}\right] \epsilon^{-2}\left(g^{R}\right)^{-1}
$$

\section{Discussion And Conclusion}

In the preceding section we have been able to derive the most generic form for the Projective real geodesics. While Theorem 2.2 describes the said geodesic over complex and conjugate coordinates, in Theorem 2.5 we've been able to arrive at a much more useful expression for the geodesic in terms of real and imaginary coordinates. Through the results, we have seen that in general the derived projective geodesic deviates from the true geodesic on the real coordinates, and indeed contains additional contributions to the 'Force field'. Careful examination of these additional terms reveal that they vanish when the underlying metric is real symmetric and the manifold itself can be locally mapped to the Minkowski spacetime. For these Gravitational systems it is shown that the Projective geodesic is identical to the true geodesic prescribed by General Relativity.

Similarly, in presence of an anti-symmetric imaginary component of the metric, 
we have seen that Theorem 2.5 naturally yields additional terms. When the imaginary component of the metric is relatively small in magnitude compared to the real part, these additional terms are shown to manifest as familiar Lorentz field with 4potential being a function of the metric itself. This is a remarkable result because unlike the existing relativistic approaches to Electromagnetism, it doesn't merely show that Lorenz Field is compatible with GR, but rather it is a manifestation of the spacetime itself, much like gravity.

The implications of Theorem 2.5 really becomes interesting and breaks into new ground for the case when both real and imaginary parts of the metric are of comparable scale. Physically speaking, these are the scenarios where there are strong enough interaction between matter and radiation. In that case, the coefficient terms $\left(g_{\mu \bar{\gamma}}^{R}+\epsilon_{\gamma}^{\eta} g_{\mu \bar{\eta}}^{I}\right)$ on the left hand side of Theorem 2.5 when inverted produces an infinite sequence of higher order terms involving $\epsilon_{\gamma}^{\eta}$. However, the form of such perturbation terms depends on the magnitude of the coupling tensor.

The scenario where $\|\epsilon\|<1$ represents relatively weak field coupling and then the perturbation series is dominated by the gravity field with Lorentz field appearing as the first order correction. However when the field interactions are stronger i.e. $\|\epsilon\| \geq 1$, gravity field vanishes and is replaced by a base term that's proportional to $\epsilon^{-2}$. In other words, for the strong coupling the dominant field diminishes with increased coupling strength and in fact for $\epsilon \rightarrow \infty$, as per Corollary[Strong Coupling], $D^{\mathbf{x}} \rightarrow 0$.

This is particularly insightful for the stability of so called charged point particles, as closer to the point, coupling becomes strong enough and inverse square law of classical electrodynamics ceases to apply.

Another encouraging aspect of the results is that it places gravity and electrodynamics on an equivalent footing emerging from a single metric connection. Since we know that Lorentz field can be quantised as Yang-Mills Gauge field, it is natural to investigate whether the same can be possible for gravity using an underlying complex manifold. This would indeed need to be explored separately and in a subsequent work.

\section{FUNDING}

Not Applicable 


\section{Conflicts of Interest}

It is hereby confirmed that there are no conflicts of interest in potential publication of the manuscript.

\section{Availability of Data And Material}

Data sharing not applicable to this article as no datasets were generated or analysed during the current study.

\section{Code Availability}

\section{Not Applicable}

\section{ReFERENCES}

[1] A. Einstein and R.W. Lawson. Relativity: The Special and the General Theory: a Popular Exposition. Bonanza paperback. Crown Publishers, 1961.

[2] H. Friedrich. Is general relativity 'essentially understood'? Annalen der Physik, 15(1-2):84-108, 2006.

[3] M.V. Berry. Principles of Cosmology and Gravitation. Taylor \& Francis, 1989.

[4] Friedrich W. Hehl, Paul von der Heyde, G. David Kerlick, and James M. Nester. General relativity with spin and torsion: Foundations and prospects. Rev. Mod. Phys., 48:393-416, Jul 1976.

[5] Donald E. Neville. Gravity theories with propagating torsion. Phys. Rev. D, 21:867-873, Feb 1980.

[6] Mark P. Haugan and C. Lämmerzahl. Principles of equivalence: their role in gravitation physics and experiments that test them. Lect. Notes Phys., 562:195-212, 2001.

[7] John Norton. What was einstein's principle of equivalence? Studies in History and Philosophy of Science Part A, 16(3):203 - 246, 1985.

[8] M. F. Atiyah and R. Bott. The yang-mills equations over riemann surfaces. Philosophical Transactions of the Royal Society of London. Series A, Mathematical and Physical Sciences, 308(1505):523615, 1983.

[9] M.E. Peskin and D.V. Schroeder. An Introduction to Quantum Field Theory. Advanced book classics. Avalon Publishing, 1995.

[10] M. Eidemüller, H.G. Dosch, and M. Jamin. The field strength correlator from qcd sum rules. Nuclear Physics B - Proceedings Supplements, 86(1-3):421-425, Jun 2000.

[11] Oskar Klein. Quantentheorie und fünfdimensionale relativitätstheorie. Zeitschrift für Physik, 37(12):895-906, 1926.

[12] Ezra T. Newman. Maxwell's equations and complex minkowski space. Journal of Mathematical Physics, 14(1):102-103, 1973.

[13] A. Trautman and Hermann Bondi. Analytic solutions of lorentz-invariant linear equations. Proceedings of the Royal Society of London. Series A. Mathematical and Physical Sciences, 270(1342):326-328, 1962.

[14] Albert Einstein. A generalization of the relativistic theory of gravitation. Annals of Mathematics, 46(4):578-584, 1945. 
[15] Albert Einstein. A generalization of the relativistic theory of gravitation. Annals of Mathematics, 46(4):578-584, 1945.

[16] Hermann Weyl. Elektron und gravitation. i. Zeitschrift für Physik, 56(5):330-352, 1929.

[17] Wolfgang Trageser. Gravitation und Elektrizität, pages 239-252. Springer Berlin Heidelberg, Berlin, Heidelberg, 2018.

[18] Hsin P. Soh. Theory of gravitation and electromagnetism. Journal of Mathematics and Physics, 12(1-4):298-305, 1933.

[19] N. Straumann. On pauli's invention of non-abelian kaluza-klein theory in 1953, 2000.

[20] J. F. Plebañski. Some solutions of complex einstein equations. Journal of Mathematical Physics, 16(12):2395-2402, 1975.

[21] Mark Davidson. A study of the lorentz-dirac equation in complex space-time for clues to emergent quantum mechanics. Journal of Physics: Conference Series, 361:012005, may 2012.

[22] Johan Bengtsson. Generalized nordström theory revisited part ii: Nordström and maxwell united, 2018.

[23] John Neumann. Mathematical foundations of quantum mechanics. Princeton University Press Princeton University Press, Princeton, 2018.

[24] Gerard't Hooft. The evolution of quantum field theory: From qed to grand unification. The Standard Theory of Particle Physics, page 1-27, Aug 2016.

[25] Victor F. Weisskopf. The development of field theory in the last 50 years. Physics Today, 34(11):69-85, November 1981.

[26] Kristian Camilleri. Constructing the myth of the copenhagen interpretation. Perspectives on Science, 17(1):26-57, 2009.

[27] Lev Vaidman. Many-worlds interpretation of quantum mechanics. In Edward N. Zalta, editor, The Stanford Encyclopedia of Philosophy. Metaphysics Research Lab, Stanford University, fall 2018 edition, 2018.

[28] Maximilian Schlosshauer, Johannes Kofler, and Anton Zeilinger. A snapshot of foundational attitudes toward quantum mechanics. Studies in History and Philosophy of Science Part B: Studies in History and Philosophy of Modern Physics, 44(3):222 - 230, 2013.

[29] E. Joos and H. D. Zeh. The emergence of classical properties through interaction with the environment. Zeitschrift fur Physik B Condensed Matter, 59(2):223-243, June 1985.

[30] Wojciech H. Zurek. Decoherence, einselection, and the quantum origins of the classical, 2001.

[31] H. D. Zeh. Decoherence: Basic concepts and their interpretation, 1995.

[32] R. Penrose. Twistor algebra. Journal of Mathematical Physics, 8(2):345-366, 1967.

[33] R. Penrose and M. A. H. MacCallum. Twistor theory: An approach to the quantisation of fields and space-time. Physics Reports, 6(4):241-315, February 1973.

[34] Lionel Mason and David Skinner. Dual superconformal invariance, momentum twistors and grassmannians. Journal of High Energy Physics, 2009(11):045-045, Nov 2009.

[35] N. Arkani-Hamed, F. Cachazo, C. Cheung, and J. Kaplan. A duality for the s matrix. Journal of High Energy Physics, 2010(3), Mar 2010.

[36] David Skinner. Twistor strings for $\mathrm{n}=8$ supergravity, 2013.

[37] Edward Witten. Perturbative gauge theory as a string theory in twistor space. Communications in Mathematical Physics, 252(1-3):189-258, Oct 2004.

Email address: swagatam.sen@gmail .com 